\title{
Biological Activities of Lactobacillus casei through the Regulation of Host Immune Function
}

\author{
Takeshi Matsuzaki
}

(Yakult Central Institute for Microbiological Research)

\begin{abstract}
Lactobacillus casei strain Shirota (LcS) has been demonstrated to have beneficial effects in numerous disease models, especially murine autoimmune disease and carcinogenesis models, via host immune modulation. It has also been reported that LcS induced recovery of the host immune responses that were decreased by treatment with carcinogens, and that augmented the natural killer activity and $T$ cell functions of host immune cells. After LcS is ingested by the host, it is incorporated into $\mathrm{M}$ cells in Peyer's patches (PP) and digested to form active components. In PP, macro-
\end{abstract}

phages or dendritic cells that phagocytosed LcS gained ability to produce tumor necrosis factor- $\alpha$. The components of LCS digested in PP were then recognized through toll-like receptor 2 in macrophages, resulting in the production of several cytokines which elicit varied responses in host immune cells. Also, it was observed by 2D-PAGE analyses that the expression level and/or the phosphorylation of some proteins in $\mathrm{PP}$ and mesenteric lymph nodes were definitely altered after the ingestion of LcS, providing more evidence of cellular responses.

\section{Screening and Development of Unique Microbial Functions and Their Industrial Applications}

\author{
Sakayu Shimizu
}

(Division of Applied Life Sciences, Graduate School of Agriculture, Kyoto University)

Over the past decade, the industrial use of microbial functions, such as their unique enzyme systems, catalysis and so on, has developed rapidly and is gathering increasing attention, particularly their use in solving environmental problems. Here, several unique microbial enzymes or reactions recently discovered in our laboratory and now used industrially are introduced, through which I will emphasize importance of screening for potential microorganisms and mutual collaboration between academia and industries.

Screening is a key step in process development, because, in many cases, the substrates in industrial processes are artificial compounds, and enzymes known to catalyze suitable reactions for such processes are still unknown. Therefore, screening for novel enzymes that are capable of catalyzing new reactions is constantly needed. In addition, the discovery of new enzymes sometimes provides clues for designing new enzymatic processes. One of the most efficient and successful means of finding new enzymes is to screen a large number of microorganisms, because of their characteristic diversity and versatility. However, it is obviously very difficult to propose rational method of screening for novel enzymes; it is something like midnight-walk without moonlight. There are three important stages in a general strategy: (1) designing the process and deciding the type of enzymatic activity desired; (2) deciding which groups of microorganisms are to be selected and screened; and (3) designing an appropriate, convenient and sensitive assay that will allow as many microorganisms as possible to be screened. It is also important, during the course of screening, to observe the functions of microorganisms carefully in order to obtain the desired (but serendipitous or random) result.

\section{Single Cell Oil Production by Mortierella alpina}

A filamentous fungus, Mortierella alpina 1S-4, was isolated as an potential producer of triacylglycerol containing C20 polyunsaturated fatty acids (PUFAs) such as arachidonic acid. With this discovery as a starting point, we conducted employing methods from metabolic engineering and molecular biology for controlling cultures and breeding mutant strains. These parental and mutant strains are now used for large-scale production of a variety of PUFAs. 
Lactonase Process for the Optical Resolution of Racemic Pantolactone

The process involves stereospecific hydrolysis step with a novel enzyme "lactonohydrolase" as the catalyst for the resolution of DL-pantolactone (DL-PL). The enzyme obtained from Fusarium oxysporum specifically hydrolyzes D-PL to D-pantoic acid (D-PA); thus DL-PL can be separated into D-PA and L-PL. When $700 \mathrm{~g} / \mathrm{L}$ aqueous solution of DL-PL was incubated with the fungal mycelia as the catalyst, D-PL in the racemic mixture was almost completely hydrolyzed to D-PA (98\%e.e.). The mycelia were immobilized into calcium alginate gels for the practical purpose. When the gels were incubated in $350 \mathrm{~g} / \mathrm{L}$ of DL-PL for $21 \mathrm{~h}$ at $30^{\circ} \mathrm{C}$ with automatic $\mathrm{pH}$ control ( $\mathrm{pH} 7.0$ ), 90-95\% of the D-PL was hydrolyzed. The resultant D-PA in the reaction mix. ture had a high optical purity (91-95\%e.e.) and the L-PL remained was unmodified. After 180 reaction cycles (i.e., 180 days), the gels retained about $60 \%$ of their initial activity.

Based on these results, the commercial production of D-PL has started since 1999, through which it was been shown that the present process is highly satisfactory not only economically but also environmentally (water $-49 \%, \mathrm{CO}_{2}-30 \%$, and $\mathrm{BOD}-62 \%$, comparing the former chemical resolution method).

\section{Bioreduction System for Large-scale Production of Chiral Alcohols}

We developed a novel bioreduction system, in which
E. coli transformant cells coexpressing an $\mathrm{NAD}(\mathrm{P}) \mathrm{H}$ dependent carbonyl reductase gene and that of glucose dehydrogenase (or formate dehydrogenase) as a cofactor regenerator are used as a catalyst, for asymmetric reduction of prochiral carbonyl compounds to chiral alcohols. Production of chiral 4-chloro-3-hydroxybutanoate esters (CHBE) is a ty pical successful example of this bioreduction system. Aldehyde reductase of Sporobolomyces salmonicolor and carbonyl reductase (S1) of Candida magnoliae were found to catalyze NADPHdependent stereospecific reduction of 4-chloro-3oxobutanoate esters (COBE) to $(R)$ - and $(S)$-CHBE, respectively, and they were successfully applied to the coexpression system for the practical production. 300$350 \mathrm{~g} / \mathrm{L}$ of $(R)$ - or $(S)$-CHBE of high optical purity (92$100 \%$ e.e.) was stoichiometrically obtained on incubation of the transformant cells in a reaction mixture containing COBE, glucose and a catalytic amount of NADP.

This bioreduction system is applicable to the production of many other useful chiral alcohols, by replacing the carbonyl reductase gene for other appropriate reductase genes. For this purpose, we have obtained various kinds of carbonyl reductases showing different substrate- and stereo-specificities through screening. Expression of the genes encoding these enzymes together with cofactor regenerator gene in E. coli cells (or other host cells) has been shown to be useful for largescale production of various kinds of chiral alcohols.

\section{Microbial Biotechnology and Future Applications}

Roy H. Dor

(Section of Molecular \& Cellular Biology, University of California, Davis)

Microbial biotechnology will play a significant role in solving many of the problems that are facing the world. Ten major problems that are challenging the world have been discussed recently at a meeting in Copenhagen, Denmark and among the Copenhagen Consensus are three problems that are relevant to microbiologists (Table 1). These challenges are (a) Communicable Diseases, (b) Malnutrition and Hunger, and (c) Sanitation and Water. In these three areas of major concern, microbiologists will be able to contribute significantly in the future and play a role by application of current and future microbiological technologies. What types of technologies will be applied in the future?
Table 1. The 10 Challenges of the Copenhagen Consensus

Armed Conflict

Climate Change

- Communicable Diseases

Education

Financial Stability

Governance and Corruption

- Malnutrition and Hunger

Population and Migration

- Sanitation and Water

Subsidies and Trade Barriers

From: J. Bohannon: Science, 304, $1429(2004)^{11}$ 